\title{
Resonant Rayleigh scattering of exciton-polaritons in multiple quantum wells
}

\author{
Malpuech, Guillaume; Kavokin, Alexey; Langbein, Wolfgang Werner; Hvam, Jørn Märcher
}

Published in:

Physical Review Letters

Link to article, DOI:

10.1103/PhysRevLett.85.650

Publication date:

2000

Document Version

Publisher's PDF, also known as Version of record

Link back to DTU Orbit

Citation (APA):

Malpuech, G., Kavokin, A., Langbein, W. W., \& Hvam, J. M. (2000). Resonant Rayleigh scattering of excitonpolaritons in multiple quantum wells. Physical Review Letters, 85(3), 650-653.

https://doi.org/10.1103/PhysRevLett.85.650

\section{General rights}

Copyright and moral rights for the publications made accessible in the public portal are retained by the authors and/or other copyright owners and it is a condition of accessing publications that users recognise and abide by the legal requirements associated with these rights.

- Users may download and print one copy of any publication from the public portal for the purpose of private study or research.

- You may not further distribute the material or use it for any profit-making activity or commercial gain

- You may freely distribute the URL identifying the publication in the public portal 


\title{
Resonant Rayleigh Scattering of Exciton-Polaritons in Multiple Quantum Wells
}

\author{
Guillaume Malpuech and Alexey Kavokin \\ Laboratoire des Sciences et Matériaux pour l'Electronique, et d'Automatique, UMR 6602 du CNRS, \\ Université Blaise Pascal-Clermont-Ferrand II, 63177 Aubière Cedex, France \\ Wolfgang Langbein \\ Lehrstuhl für Experimentelle Physik EIIb, Universität Dortmund, Otto-Hahn Strasse 4, 44227 Dortmund, Germany \\ Jørn M. Hvam \\ Research Center COM, Technical University of Denmark, Building 349, DK-2800 Lyngby, Denmark
}

(Received 25 October 1999)

\begin{abstract}
A theoretical concept of resonant Rayleigh scattering (RRS) of exciton-polaritons in multiple quantum wells (QWs) is presented. The optical coupling between excitons in different QWs can strongly affect the RRS dynamics, giving rise to characteristic temporal oscillations on a picosecond scale. Bragg and anti-Bragg arranged QW structures with the same excitonic parameters are predicted to have drastically different RRS spectra. Experimental data on the RRS from multiple QWs show the predicted strong temporal oscillations at small scattering angles, which are well explained by the presented theory.
\end{abstract}

PACS numbers: 78.35. $+\mathrm{c}, 78.47 .+\mathrm{p}, 78.66 .-\mathrm{w}$

The essential difference between the resonant Rayleigh scattering [1] (RRS) and the photoluminescence (PL) is that the optical coherence is kept in the former case and broken in the latter case, which is not easily distinguished experimentally. Recently, RRS by excitons in semiconductor quantum wells (QWs) has been reported $[2,3]$. The spectroscopic data have been interpreted in terms of scattering of excitons by a disorder potential in the plane of the QWs [4-6]. Strictly speaking, this interpretation contains a controversy, since the bare excitons [7] themselves are not coherent with the incident light. Actually, they are formed as a result of dissociation of exciton-polaritons with a loss of coherence. That is why the bare excitons govern the PL spectra, while to describe the RRS spectra one should speak in terms of the exciton-polaritons. This difference is extremely important in multiple quantum well (MQW) structures where the bare excitons are localized in the individual QWs, but the exciton-polaritons occupy the entire structure. Recently, there was a series of papers that aimed to reveal the role of exciton-polaritons in $\mathrm{cw}$ and time-resolved reflection of MQWs [8]. Moreover, Rabi oscillations in the RRS spectra of microcavities have been reported recently [9], which is a purely polaritonic effect.

This Letter aims to show that the RRS spectra of MQWs are a result of exciton-polariton scattering involving all the QWs. In particular, the RRS signal is sensitive to the period of the MQW structure and the number of QWs. Especially Bragg- and anti-Bragg-arranged MQWs exhibit drastically different RRS spectra. Experimental data on MQWs with small interwell spacing are presented. Strong temporal oscillations of the RRS are observed for small scattering angles in a very good agreement with the presented theory.

The previous studies of the RRS by MQWs can be briefly summarized as follows. The data reported in
Ref. [2] exhibit typically a RRS signal that initially rises quadratically with time and then decays nonexponentially on the time scale of the inverse inhomogeneous broadening of the exciton peak. This fast decay is followed by a slower exponential decay. The rise and the fast initial decay have been explained by a theoretical model [5] which assumes an ensemble of classical oscillators moving within a random potential with a finite correlation length. Recent experiments on different MQW structures have demonstrated a complex nonmonotonic decay sometimes showing oscillations with a period of a few picoseconds [4]. These oscillations have been associated in Ref. [4] to the interference between light waves scattered by different localized exciton states in a QW plane. Experiments on single QWs [3] do not show such features and are well explained using a correlation length shorter than the exciton radius. Reference [10] has demonstrated a possibility of the secondary emission (SE) from QWs due to the quantum fluctuations of the light field. This theory written for an ideal single QW has no direct relation to the discussed experiments performed in the linear regime on the a priori disordered MQW samples. An attempt to take into account the polariton effects in the RRS has been done by Citrin [11]; however, all presented numerical calculations ignored the polariton effect, so that their role, especially in the RRS of MQWs, remained unclear.

The observed discrepancies between the RRS from SQWs and MQWs point out the need for an excitonpolariton theory, which requires a revision of the physical concept of the RRS in QWs, namely, introducing the concept of scattering of coherent exciton-polariton states.

In our approach, the dielectric response of QWs containing inhomogeneously broadened exciton resonances is described in the framework of the nonlocal model [12]. The parameters used for the individual QWs are the 
exciton radiative damping rate $\Gamma_{0}$, the nonradiative homogeneous broadening $\gamma$, and the Gaussian inhomogeneous broadening $\Delta$. For a MQW structure, we index by $z_{j}$ the coordinate of each QW and calculate the electric fields $E_{g}\left(z_{j}, \omega\right)=g(\omega) E\left(z_{j}, \omega\right)$ (with $j$ varying from 1 to $N$ ), where $E(z, \omega)$ is the stationary solution of the Maxwell equations corresponding to a plane light-wave incident on the structure from the left, and $g(\omega)$ is the spectral function of the incident pulse. In all further calculations we assume the incident pulse of light to be 500 fs long with a center frequency at the exciton resonance.

The probability to create an exciton having a frequency $\omega$ and a wave vector $k_{\|}=0$ in the $j$ th $\mathrm{QW}$ is proportional to the electric field intensity in this well. The amplitude of the electric field of the scattered exciton-polariton state with $k_{\|} \neq 0$ can then be found using Eq. (28) of Ref. [6]:

$$
A_{s}\left(z_{j}, \omega\right)=P_{s}(\omega) E_{g}\left(z_{j}, \omega\right),
$$

where

$$
\begin{aligned}
P_{s}(\omega)= & \int_{-\infty}^{+\infty}\left(\int\left\{\exp \left[\left(t / t_{0}\right)^{2} f_{R}\right]-1\right\} d R\right)^{1 / 2} \\
& \times \exp \left[-\left(t / \sqrt{2} t_{0}\right)^{2}\right] \exp (i \omega t) d t
\end{aligned}
$$

with the rise time $t_{0}=\sqrt{2} \hbar / \Delta$ and $f_{R}$ being a potential correlation function chosen in form:

$$
f_{R}=\exp \left(-R^{2} / 2 \xi^{2}\right),
$$

where $\xi$ is the potential correlation length further assumed to be $\xi=5 \mathrm{~nm}$. Note that Eq. (2) is written in the classical limit. The time-dependent amplitude of the scattered light in the right part of Eq. (2) is real, while its Fourier transform $P_{s}(\omega)$ is a complex function. No random phase fluctuations are taken into account.

The scattered amplitudes can be considered as light emitted by each well. Using the generalized scatteringstates method [13], we calculate their propagation in the scattering direction. Let us consider an emitter of light situated in the center of the $j$ th QW. We neglect the spatial distribution of the emitter, which is a usual assumption in the nonlocal model. We take into account the polariton effect within each single QW, i.e., the possibility of emission and absorption of photon by the same exciton. Using the transfer matrix method for a given wave vector of light $k_{\|}$, in the $p$ polarization we calculate the reflection and transmission coefficients of the right part of the structure (between $z=z_{j}$ to $z=d$, where $d$ is the total length of the MQW structure), labeled $r_{r}^{j}$ and $t_{r}^{j}$, respectively, as well as those of the left part of the structure (between $z=z_{j}$ to $z=0$ ), labeled $r_{l}^{j}$ and $t_{l}^{j}$, respectively.

Formally, we consider our structure as a Fabry-Pérot resonator with an infinitely thin central layer, where the emitter is placed. Taking into account all multiple reflections in the resonator, we can write the electric field just to the right from the source as

$$
\tilde{E}_{+}^{z_{j},+}\left(\omega, z_{j}\right)=\frac{1}{1-r_{r}^{j} r_{l}^{j}},
$$

and the field just to the left from the emitter as

$$
\tilde{E}_{-}^{z_{j},+}\left(\omega, z_{j}\right)=\frac{r_{r}^{j}}{1-r_{r}^{j} r_{l}^{j}} .
$$

We calculate the electric field at the end and at the beginning of the entire structure as

$$
\begin{aligned}
& \tilde{E}_{+}^{z_{j},+}(\omega, d)=\frac{t_{r}^{j}}{1-r_{r}^{j} r_{l}^{j}}, \\
& \tilde{E}_{-}^{z_{j},+}(\omega, 0)=\frac{r_{r}^{j} t_{l}^{j}}{1-r_{r}^{j} r_{l}^{j}},
\end{aligned}
$$

respectively.

In the same way we calculate the amplitudes $\tilde{E}_{+}^{z_{j},-}\left(\omega, z_{j}\right), \quad \tilde{E}^{z_{j},-}\left(\omega, z_{j}\right), \quad \tilde{E}^{z_{j},-}(\omega, 0), \quad \tilde{E}_{+}^{z_{j},-}(\omega, d)$, in the case of a $\delta$-pulse emitted form $z=z_{j}$ in the negative direction. Here the notation is completely analogous to that used in Eqs. (4)-(7).

The amplitude of light emitted by the $j$ th QW with a spectral function $A_{s}\left(z_{j}, \omega\right)$ at the surface of the structure is

$$
\tilde{E}_{A}^{z_{j}}(\omega, 0)=A_{s}\left(z_{j}, \omega\right)\left[\tilde{E}_{-}^{z_{j},+}(\omega, 0)+\tilde{E}_{-}^{z_{j},-}(\omega, 0)\right] .
$$

Thus, the amplitude of light scattered in a given direction by the system containing $N$ QWs is

$$
r_{s}(\omega)=\sum_{j=1}^{N} \tilde{E}_{A}^{z_{j}}(\omega, 0) .
$$

The time-resolved RRS signal is given by

$$
R_{s}(t)=\left|\frac{1}{2 \pi} \int_{-\infty}^{+\infty} r_{s}(\omega) \exp (-i \omega t) d \omega\right|^{2} .
$$

In summary, we take into account all the coherent reflection-absorption processes for this scattered light in the structure. We ignore the secondary scattering, however.

Let us first compare the results of the present theory with experimental data on the RRS from single quantum wells (SQWs) and MQWs. In the following we discuss experiments performed on $\mathrm{GaAs} / \mathrm{Al}_{0.3} \mathrm{Ga}_{0.7} \mathrm{As} \mathrm{QWs}$ grown by molecular-beam epitaxy without growth interruption. The MQWs consist of ten wells and have barrier thicknesses of $15 \mathrm{~nm}$. They are placed in a helium cryostat at $5 \mathrm{~K}$ temperature. The fundamental $h h 1-e 11 s$ exciton resonance is excited by optical pulses from a mode-locked Ti:sapphire laser of about 500 fs Fourier-limited pulse duration. The SE is passed through an analyzer parallel to the linear excitation polarization ( $p$ polarization), a monochromator, and is detected time and angular resolved by a synchroscan streak camera with a time resolution of $3 \mathrm{ps}$. The angular resolution was adjusted close to a single speckle, i.e., to the diffraction limit of the excited area on the sample. The spectral resolution of about $1 \mathrm{meV}$ rejects nonresonant emission but does not deteriorate the temporal resolution. The excited exciton density of around $10^{8} \mathrm{~cm}^{-2}$ per well was close to the low density limit. Using the 
speckle analysis technique [3], we deduce from the temporally and directionally resolved emission intensity $I(t, \vec{q})$ the average emission intensity $\overline{I(t)}$ and the average coherence $c=\overline{I_{\text {coh }}} / \bar{I}$, where the average is taken over the scattering directions $\vec{q}$ at fixed time, and $I_{\text {coh }}$ is the SE intensity which is coherent to the excitation.

In Fig. $1 \overline{I(t)}$ for a $16 \mathrm{~nm}$ MQW and a $15 \mathrm{~nm} \mathrm{SQW}$ sample are shown on the left for two different scattering angles. Since the wells in both samples have nearly equal thicknesses, the inhomogeneous broadening of the individual wells due to the interface roughness is comparable (just slightly more in the SQW structure having a thinner QW). The SE linewidth (FWHM) for large scattering angles is $170 \mu \mathrm{eV}$ for the SQW and $340 \mu \mathrm{eV}$ for the MQW. The increased linewidth of the MQW is in good agreement with the calculation and is due to the radiative coupling between the wells, giving rise to ten radiative modes of different radiative broadenings and renormalizations. The nonradiative homogeneous broadening $\gamma$ is about $6 \mu \mathrm{eV}$ in both samples, as deduced from the decay of the SE coherence decay for long times. While for large scattering angles (bottom), the dynamics is qualitatively comparable in both structures, at small scattering angles (top) the MQW shows strong modulations of the RRS within the first $40 \mathrm{ps}$, which are not observed for the SQW. On the right side of Fig. 1 the calculated RRS corresponding to the measurements on the left for the exact structures of the SQW and MQW are shown, using the $\Gamma_{0}=0.02 \mathrm{meV}, \gamma=0.006 \mathrm{meV}$, and $\Delta=0.082 \mathrm{meV}$ for the MQW and $\Delta=0.10 \mathrm{meV}$ for the SQW (note that the FWHM of the Gaussian distribution is $2 \sqrt{\ln 2} \cdot \Delta$ and that all parameters are determined by experiments independent of the SE dynamics). For the small scattering

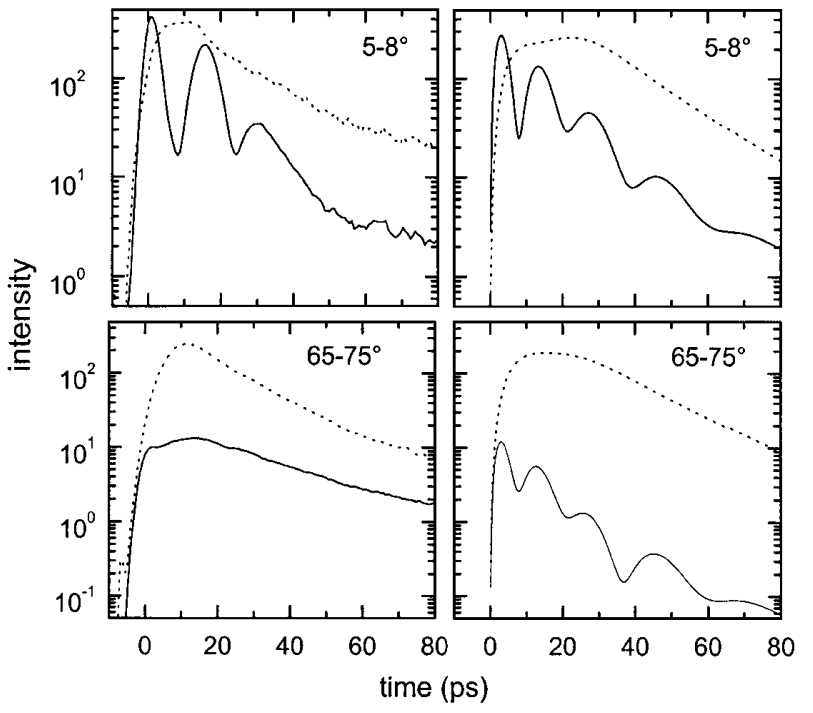

FIG. 1. Measured SE intensity (left) and calculated RRS intensity (right) versus time for a $10 \times 16 \mathrm{~nm} \mathrm{MQW} \mathrm{(solid} \mathrm{line)}$ and a $15 \mathrm{~nm} \mathrm{SQW} \mathrm{(dotted} \mathrm{line).} \mathrm{Top:} \mathrm{small} \mathrm{scattering} \mathrm{angle}$ $\left(\sim 6^{\circ}\right)$; bottom: large scattering angle $\left(\sim 70^{\circ}\right)$. angle, the calculations are in good agreement with the experimental data, confirming that the observed modulations in the MQW structure are indeed due to the polaritonic coupling and that the presented model is suitable to model the RRS of exciton-polaritons in MQWs.

For the large angle scattering, the present model predicts only a slight reduction of the modulation in the RRS, while in the experiment the modulations are strongly suppressed. This discrepancy may be associated to the higher-order scattering or to the angle dependence of the inhomogeneous broadening parameter $\Delta$. Note also that the use of a classical limit in Eq. (2) is an approximation.

An increase of the inhomogeneous broadening is expected to reduce the importance of these modulations, as in time-resolved reflection spectra [8]. We investigate this using MQWs of different well thicknesses $L$, using the fact that the inhomogeneous broadening is roughly proportional to $L^{-3}$. In Fig. 2 we show $\overline{I(t)}$ at small scattering angles from MQWs of 13,10 , and $8 \mathrm{~nm}$ well thicknesses, showing SE linewidths (FWHM) of 0.42, 0.7, and $1.1 \mathrm{meV}$, respectively. While a strong initial RRS modulation is present for all structures, the duration of this enhanced emission decreases with an increase of the inhomogeneous broadening. Model calculations are shown for comparison (dotted line). The $\Delta$ used in the calculations is determined using the experimental linewidth of the $8 \mathrm{~nm}$ MQW sample, which is dominated by the inhomogeneous broadening, and the $L^{-3}$ scaling as $\Delta=0.15,0.34$, and $0.66 \mathrm{meV}$, respectively. This is compatible with the value of $0.1 \mathrm{meV}$ used for the $15 \mathrm{~nm}$ SQW in Fig. 1. $\Gamma_{0}$ was taken to be 22,26 , and $28 \mu \mathrm{eV}$, respectively, from [14]. We also used $\gamma=0.006 \mathrm{meV}$. The initial part (up to $20 \mathrm{ps)}$ ) of all the spectra is well described by the theory (note, in particular, the doublepeak structure for the $13 \mathrm{~nm}$ MQWs). This confirms that also the influence of the inhomogeneous broadening is modeled correctly in the calculation. At longer times, the discrepancy between theory and experiment appears which is likely due to secondary scattering.

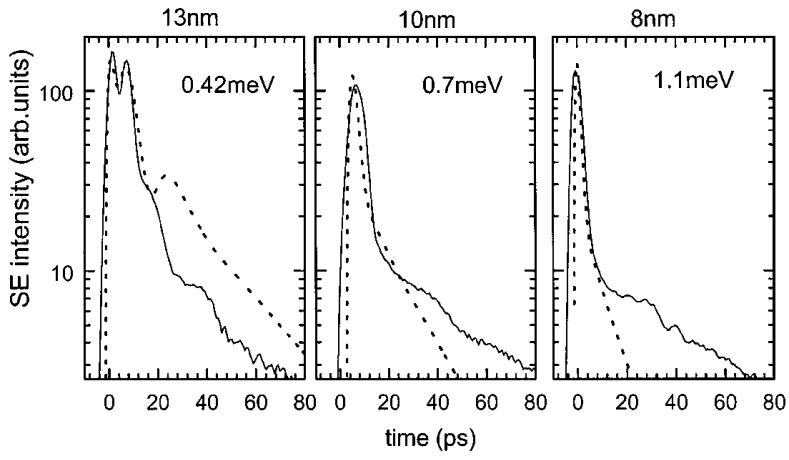

FIG. 2. Measured SE intensity (solid line) and calculated RRS intensity (dotted line) versus time for a $10 \times 13 \mathrm{~nm}$ MQW (left), $10 \times 10 \mathrm{~nm} \mathrm{MQW} \mathrm{(middle),} \mathrm{and} \mathrm{a} 10 \times 8 \mathrm{~nm} \mathrm{MQW}$ (right) for a small scattering angle $\left(\sim 6^{\circ}\right)$. 


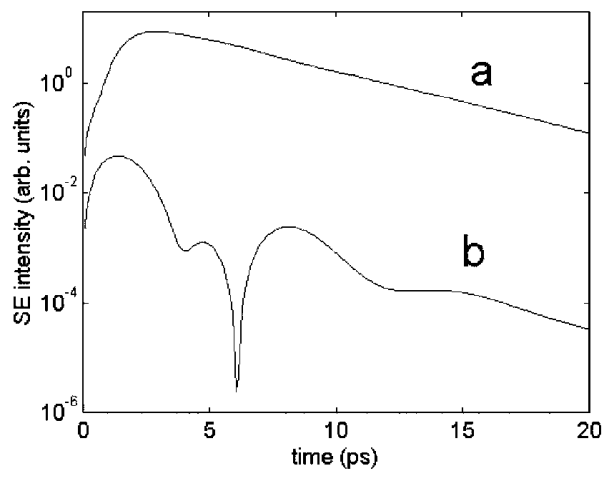

FIG. 3. Time-resolved RRS spectra calculated for 25 Braggarranged (a) and 25 anti-Bragg-arranged (b) MQWs.

The specific period of a MQW structure is strongly effecting the RRS spectra. In order to demonstrate this, let us compare the Bragg- and anti-Bragg-arranged MQW structures with the same number of QWs. In the Braggarranged MQWs the spacing between wells is equal to half a wavelength of light at the exciton resonance frequency. In this configuration, only one super-radiant excitonpolariton mode is active [15]. In the anti-Bragg-arranged QWs (spacing between wells equal to a quarter-wavelength of light at the exciton frequency) the eigenmodes are symmetrically distributed around the bare exciton-resonance frequency [16] thus allowing various quantum oscillation effects.

Figure 3 shows the RRS calculation for 25 Braggarranged and 25 anti-Bragg-arranged QWs. We assumed a GaAs $/ \mathrm{Al}_{0.3} \mathrm{Ga}_{0.7} \mathrm{As}$ structure embedded in $\mathrm{Al}_{0.3} \mathrm{Ga}_{0.7} \mathrm{As}$ barriers and the following QW exciton parameters: $\Gamma_{0}=$ $0.028 \mathrm{meV}$ (that corresponds to a QW of $8 \mathrm{~nm}$ ), $\gamma=$ $0.006 \mathrm{meV}$, and $\Delta=0.5 \mathrm{meV}$. The cap-layer thickness for both structures is $15 \mathrm{~nm}$. The incidence angle is $0^{\circ}$; the scattering at small angles (within $5^{\circ}$ ) is considered. One can see that there are no oscillations in the RRS spectra from the Bragg-arranged MQW structure. On the contrary, the RRS from the anti-Bragg-arranged MQWs exhibit pronounced irregular oscillations. These oscillations arise due to the interference between different bright polaritonic modes in the MQWs. The drastic difference between RRS spectra of Bragg and anti-Bragg-arranged QWs evidences the dominant role of exciton-polaritons in the RRS.

In conclusion, RRS from multiple quantum well structures is governed by the dynamics of the exciton- polaritons, which are extended mixed exciton-photon states occupying the entire system. The present semiclassical model takes into account the exciton-polariton effect in MQWs and describes correctly the experimental RRS dynamics from GaAs MQWs for small scattering angles, which are dominated by the exciton-polariton dynamics. Inhomogeneous broadening is found to limit the time range on which exciton-polariton dynamics can be dominating, both in experiment and simulation. Drastic differences in the time-resolved RRS of Bragg- and antiBragg-arranged MQWs are predicted.

[1] R. Loudon, The Quantum Theory of Light (Oxford Science Publications, Oxford, 1983).

[2] H. Wang et al., Phys. Rev. Lett. 74, 3065 (1995); S. Haacke et al., Phys. Rev. Lett. 78, 2228 (1997); M. Woerner and J. Shah, Phys. Rev. Lett. 81, 4208 (1998); N. Garro et al., Phys. Rev. B 60, 4497 (1999); D. Birkedal and J. Shah, Phys. Rev. Lett. 81, 2372 (1998).

[3] W. Langbein, J. M. Hvam, and R. Zimmermann, Phys. Rev. Lett. 82, 1040 (1999); W. Langbein and J. M. Hvam, in Advances in Solid State Physics (Vieweg, Braunschweig, 1999), Vol. 39, p. 463.

[4] V. Savona, S. Haacke, and B. Deveaud, Phys. Rev. Lett. 84, 183 (2000).

[5] R. Zimmermann, Nuovo Cimento Soc. Ital. Fis. 17D, 1801 (1995).

[6] V. Savona and R. Zimmermann, Phys. Rev. B 60, 4928 (1999).

[7] V. M. Agranovich and V.L. Ginzburg, Crystal Optics with Spatial Dispersion and Excitons (Springer, Berlin, 1984).

[8] J. J. Baumberg et al., Phys. Rev. Lett. 80, 3567 (1998); G. R. Hayes et al., Phys. Rev. Lett. 83, 2837 (1999); M. Hübner et al., Phys. Rev. Lett. 83, 2841 (1999).

[9] G. R. Hayes et al., Phys. Rev. B 58, R10 175 (1998).

[10] M. Kira, F. Jahnke, and S. W. Koch, Phys. Rev. Lett. 82, 3544 (1999).

[11] D. S. Citrin, Phys. Rev. B 54, 14572 (1996).

[12] L. C. Andreani et al., Phys. Rev. B 57, 4670 (1998).

[13] G. Malpuech, A. Kavokin, and G. Panzarini, Phys. Rev. B 60, 16788 (1999).

[14] L. C. Andreani and A. Pasquarello, Phys. Rev. B 42, 8928 (1990).

[15] E. L. Ivchenko, A. I. Nesvizhski, and S. Jorda, Phys. Solid State 36, 1156 (1994).

[16] M. R. Vladimirova, E.L. Ivchenko, and A. V. Kavokin, Semiconductors 32, 90 (1998). 